\title{
A THEOREM ON THE AFFINE TRANSFORMATION GROUP OF A RIEMANNIAN MANIFOLD
}

\author{
SHOSHICHI KOBAYASHI
}

\section{Introduction}

Every Riemannian manifold has a unique affine connection without torsion, which is necessarily invariant by any isometrical transformation of the manifold. However, an affine transformation (i.e., transformation leaving invariant the affine connection) is not necessarily an isometrical transformation. (Consider, for example, the ordinary Euclidean space).

Let us denote by $M$ an $n$-dimensional Riemannian manifold and by $I(M)$ (resp. $A(M)$ ) the group of all isometrical (resp. affine) transformations of $M$. Then, in general, $I(M)$ is a closed subgroup of $A(M)$. Yano [2] proved that, if $M$ is compact, then the connected component of the unit of $A(M)$ is contained in $I(M)$. Nomizu [1] obtained also some results concerning the relation between $A(M)$ and $I(M)$. The purpose of the present paper is to prove the following theorem : ${ }^{1)}$

Theorem. If $M$ is an irreducible and complete Riemannian manifold, then $A(M)$ is equal to $I(M)$, except the case $M$ is the 1-dimensional Euclidean space.

\section{Reduction of the problem}

Let $d s^{2}$ be the Riemannian metric on $M$, which we are considering. If we denote by $\varphi^{*}\left(d s^{2}\right)$ the metric induced by an affine transformation $\varphi$, then we have the following lemma: [1]

Lemma. If $M$ is irreducible, then $\varphi^{*}\left(d s^{2}\right)=c^{2} \cdot d s^{2}$, where $c$ is a positive constant.

Therefore we have only to show that the constant $c$ is equal to 1 . The

Received March 21, 1955.

* This theorem has been proved independently by $T$. Nagano for the connected component of $A(M)$, using the theory of infinitesimal affine transformations and Killing vector fields (not yet published). J. Hano has also proved a similar result which generalizes the theorem of Yano (see his paper in this journal). 
proof will be divided into two cases:

(1) $\varphi$ has no fixed point.

(2) $\varphi$ has a fixed point.

\section{The first case}

Suppose that an affine transformation $\varphi$ of $M$ has no fixed point and that the constant $c$ is different from 1 . Clearly the inverse transformation of $\varphi$ has no fixed point; hence we may assume that the constant $c$ is less than 1 . Take an arbitrary point $x$ in $M$ and a geodesic $g$ joining $x$ and $\varphi(x)$. Then $\varphi^{k}(g)$ is a geodesic joining $\varphi^{k}(x)$ and $\varphi^{k+1}(x)$. If $d$ is the length of the geodesic $g$, then the length of the geodesic $\varphi^{k}(g)$ is $c^{k} d$. Therefore the points $\varphi^{k}(x), 0 \leqq$ $k<\infty$, form a Cauchy sequence. Since $M$ is complete, this sequence converges to a point $x^{*}$. Then it is easy to see that the point $x^{*}$ is left fixed by $\varphi$. Now we have proved that, if an affine transformation $\varphi$ of an irreducible and complete Riemannian manifold $M$ has no fixed point, then it is an isometry.

This result is true even for the 1-dimensional Euclidean space.

\section{The second case}

Suppose that an affine transformation has a fixed point $x$ and that the constant $c$ is different from 1 . We may assume again $c<1$.

Let $\gamma$ be a piecewise differentiable closed curve in $M$ starting from $x$. Since $c<1$, the sequence of curves $\varphi^{k}(\gamma)$ converges to the point $x$. Furthermore the first derivatives (for the values of parameter for which the curves are differentiable) of curves $\varphi^{k}(\gamma)$ tend to zero, because the curves $\varphi^{k}(\gamma)$ are shortened uniformly when $k$ tends to the infinity. Let $h_{k}$ be the element of the homogeneous holonomy group associated to the closed curve $\varphi^{k}(\gamma)$; it is an orthogonal transformation of the tangent space $T_{x}$ to $M$ at $x$. Then $h_{k}$ tends to the identity transformation when $k$ tends to the infinity.

Let $\delta \varphi$ be the linear transformation of $T_{x}$ induced by $\varphi$. Since $\varphi$ is an affine transformation we haye

$$
h_{0}=\delta \varphi^{-1} h_{1} \cdot \delta \varphi
$$

By induction we obtain

$$
h_{0}=\delta \varphi^{-k} h_{k} \cdot \delta \varphi^{k}
$$


Let $P_{k}$ be the characteristic polynomial of the transformation $\delta \varphi^{-k} h_{k} \cdot \delta \varphi^{k}$, which is equal to the characteristic polynomial of $h_{k}$. As $h_{k}$ tends to the identity transformaton, the $P_{k}$ tends to the following polynomial:

$$
(1-\lambda)^{n}
$$

where $\lambda$ is the variable of the polynomial and $n$ is the dimension of $M$. Since the characteristic polynomial of $h_{0}$ is equal to all the polynomials $P_{k}$, it must be $(1-\lambda)^{n}$. Hence $h_{0}$ is the identity transformation of the tangent space $T_{x}$. Now we have proved that, for any closed curve $r$ starting from $x$, the element of the homogeneous holonomy group associated to $\gamma$ is the identity transformation.

Hence $M$ is locally Euclidean (in the sense of differential geometry). As $M$ is complete and irreducible, it must be the 1-dimensional torus or the 1-dimensional Euclidean space. If it is the 1-dimensional torus, then it is easy to see that $A(M)=I(M)$. This completes the proof of the theorem.

\section{BiBLIOGRAPHY}

[1] Nomizu, K.: Sur les transformations affines d'une variété riemanniennes. C. R. Acad. Paris, 237 (1953), 1308-1310. Also, Studies on Riemannian homogeneous spaces (in this journal).

[2] Yano, K.: On harmonic and Killing vector fields. Ann. Math. 55 (1952), 38-45.

University of Washington 